\title{
Auxiliary ischemic markers, the role of left ventricular dyssynchrony
}

\author{
Teresa Massardo, $M^{a}$ \\ a Sección Medicina Nuclear, Departamento de Medicina, Hospital Clínico Universidad de Chile, \\ Santiago, Chile
}

Received Jan 10, 2019; accepted Jan 11, 2019

doi: $10.1007 / \mathrm{s} 12350-019-01606-1$

\section{See related article, pp. 2261-2268}

Myocardial ischemia due to coronary artery disease (CAD) could be detected with a high sensitivity using available imaging methods. However, even with stress single photon emission tomography (SPECT) myocardial perfusion imaging (MPI) ischemia may not be detected in some patients.

Several indirect parameters have been considered in addition to the perfusion pattern, such as stress-transient ischemic dilation (TID), stress right ventricular perfusion, change (rest-stress) left ventricular ejection fraction (LVEF) and transient segmental wall motion and thickening abnormalities. ${ }^{1}$

Left ventricular (LV) mechanical contraction synchrony has been proposed as a promising tool for selecting chronic heart failure patients who could respond better to resynchronization therapy. ${ }^{2-4}$ It has been advocated also as a useful marker for ischemia detection, although the results might be affected by several factors such as tracer selection, timing of stress acquisition (early or delayed) or type of stress employed (exercise or vasodilator).

Gender seems also to affect the results: in a normal Japanese database, males presented greater variation in phase histogram parameters; counts and cardiac size could influence the findings according to the authors. ${ }^{5}$ Moreover, the type of protocol and its sequence (by instance: one-day, stress first) should also be considered.

Reprint requests: Teresa Massardo, MD, Sección Medicina Nuclear, Departamento de Medicina, Hospital Clínico Universidad de Chile, Santos Dumont 999 1E, Independencia, PO Box 6531063, Santiago, Chile; teresamassardo4@gmail.com

J Nucl Cardiol 2020;27:2269-72.

$1071-3581 / \$ 34.00$

Copyright (C) 2019 American Society of Nuclear Cardiology.
The number of frames used for gating (8 vs 16) do not appear to affect the results ${ }^{6}$; however, conduction abnormalities such as left bundle branch block or pacemaker rhythm alters the measurement. Proper myocardial wall detection is critical for repeatability and reproducibility with automated software, but a careful manual adjustment of the limits, especially at the LV base is often needed. ${ }^{7}$ Injected activity matters as well in this type of analysis; LV mechanical dyssynchrony indices by phase analysis have more variation and are significantly higher if derived from gated SPECT with low-dose radiotracer. ${ }^{8}$ In a very recent report, heart rate has also been mentioned as important affecting results; time measurement using QGS phase analysis in milliseconds rather than degrees of the cardiac cycle is preferred in patients with normal perfusion. ${ }^{9}$

Normal myocardium contracts with a narrow synchrony histogram at rest $^{3}$; with stress, the histogram becomes narrower, due to more efficient muscular contraction observed better when using exercise and early imaging. This is also detected with $\mathrm{Rb}-82$ positron emission tomography (PET) imaging independent of the resting LVEF. ${ }^{10}$ Tl-201 SPECT MPI early post-stress acquisition can demonstrate LV mechanical dyssynchrony assessed by phase analysis. ${ }^{11,12}$ In a study with multi-vessel CAD patients using Tl-201 and dipyridamole, more global and territorial dyssynchrony at early post-stress than at rest was observed. ${ }^{13}$ A case with severe and extensive CAD using Tl-201 with dipyridamole demonstrated incremental value for ischemic stunning detection. ${ }^{14}$ In our experience, when using dipyridamole, the delayed acquisition (60 minutes posttracer injection) makes ischemia detection with phase analysis more difficult, even in cases with significant reversible perfusion defects. We could not find differences between rest and post-stress synchrony in these cases. ${ }^{15}$ Others have shown that the presence of a large reversible perfusion defect does not alter mechanical dyssynchrony by phase analysis. Comparable 
information was obtained whether using low- or highdose radiotracer; the authors obtained their results from 20 patients without perfusion defects and 20 with transient defects employing 1 hour delayed stress acquisition, with exercise in only $30 \%$ of their cases. ${ }^{16}$

Another problematic issue in phase analysis is the method selected to define an abnormal LV mechanical contraction. There are different programs to measure it, such as ECTb Emory Tool Box, QGS, 4DM Corridor and cardioGRAF ${ }^{3,17-19}$ and several data bases. ${ }^{3,5}$ It appears that the Tc-99m sestamibi SPECT normal values post exercise are not different from Rb-82 PET dipyridamole ones. ${ }^{20}$

In the retrospective work presented in the current issue of the Journal, Camilletti et al provide new information regarding $\mathrm{LV}$ ischemia detection through myocardial stunning using phase analysis. Their perfusion SPECT images were acquired early post-exercise. They evaluated two groups: normal (no ischemia) and ischemia patients excluding those with SDS 1 to 5 as well as those with scar, in order to measure the extent of the ischemia and possible stunning. LV synchrony was compared with wall motion assessed visually and their gold standard was the perfusion status. Their results are in agreement with a similar recent publication using sestamibi with acquisition at 15 minutes post-exercise injection. The authors found that baseline differences in LV volumes and degree of intraventricular dyssynchrony were associated with inducible ischemia and increasing histogram bandwidth $(\mathrm{BW}){ }^{21}$ Exercise SPECT synchrony analysis was also shown to increase multivessel CAD detection when combining phase standard deviation (SD), TID ratio and perfusion summed stress score (SSS) compared with TID ratio and SSS alone. ${ }^{22}$

It is interesting to point out that ischemic dyssynchrony has been observed as well with stress dynamic PET with Rb-82 PET using regadenoson ${ }^{23}$ in normal MPI, with similar method, the indices are smaller at peak stress vs. rest, independently of LVEF at rest. ${ }^{10}$ Coronary steal described with vasodilators probably do not produce real stunning as observed with exercise or it may last for a shorter period. Visual wall motion measurement is also difficult; in a prior study by our group employing first pass technique and using sestamibi dipyridamole-isometric exercise, wall motion abnormalities were not helpful in the assessment of $\mathrm{CAD}^{24} \mathrm{~A}$ recent work on systolic wall thickening, ${ }^{25}$ described better correlation with ischemia than LVEF changes, when performing a 2 days exercise protocol with a Tc$99 \mathrm{~m}$ tetrofosmin and delayed acquisition. Wall thickening-summed difference score quantified the stunning phenomenon, being an independent parameter with the strongest correlation observed with the degree of ischemia and reversible systolic dysfunction. In a later work ${ }^{26}$; the same group published that systolic thickening with dipyridamole also showed better correlation with the degree of ischemia than with global LV function allowing a better identification of the stunning phenomenon.

Camilletti et al mention in their work that both dyssynchrony and wall motion abnormalities using exercise stress are of great value as auxiliary markers for CAD detection. However, their results like other observations demonstrate that LV wall motion is not very sensitive and that dyssynchrony is a better marker. Possibly both parameters together could be helpful considering automated motion measurement instead of visual wall analysis. In their investigation, there was not a classical gold standard available (coronary angiography) or ideally absolute myocardial flow measurement.

LV dyssynchrony evaluated by phase analysis with gated MPI may provide a good prognostic value. This was demonstrated in end-stage renal disease patients as part of their renal transplant evaluation even beyond information provided by myocardial perfusion and LVEF as predictors of all-cause mortality. ${ }^{27}$ After adjusting for demographics, co-morbidities, LVEF, and perfusion pattern, phase analysis parameters, particularly phase $\mathrm{BW}$, was independently associated with worse outcome. In that large study, exercise and vasodilator stress (adenosine) were employed and phase parameters analyzed from a stress phase acquired at rest, according to the authors. On the other hand, significant mechanical dyssynchrony have been found in gated SPECT MPI with stronger association with global and cardiovascular mortality compared with electrical dyssynchrony measured with QRS duration. ${ }^{28}$

In summary, more studies are warranted to confirm that phase analysis can add significant value over other parameters of LV perfusion and function to detect ischemia using gated SPECT MPI. This could also be important in more vulnerable population such as diabetics with cardiovascular risk factors with possible silent ischemia; cases with negative stress MPI or in those with normal stress perfusion SPECT representing balanced three-vessel disease with diffuse stunning. However, some authors believe that synchrony parameters have value only in the presence of reversible perfusion defects. ${ }^{29}$ Moreover, phase analysis could be more helpful in the group with doubtful, mild or equivocal LV ischemia (including those cases not incorporated in Camilletti's work with SSS $<5$ or those with mild SDS values not considered significant). A larger sample of patients with clinical follow-up and a robust gold standard for myocardial ischemia is still missing in this important subject. We believe that the additional value of phase analysis technique in ischemia 
detection and its prognostic value to assess intraventricular contraction with diverse stress modalities should be confirmed further when using MPI with Tc-99m tracers.

\section{Disclosures}

The author has no conflict of interest to disclose.

\section{References}

1. Nakanishi R, Gransar H, Slomka P, Arsanjani R, Shalev A, Otaki $\mathrm{Y}$, et al. Predictors of high-risk coronary artery disease in subjects with normal SPECT myocardial perfusion imaging. J Nucl Cardiol 2016;23:530-41.

2. Boogers MJ, Chen J, van Bommel RJ, Borleffs CJ, Dibbets-Schneider P, van der Hiel B, et al. Optimal left ventricular lead position assessed with phase analysis on gated myocardial perfusion SPECT. Eur J Nucl Med Mol Imaging 2011;38:230-8.

3. Chen J, Garcia EV, Folks RD, Cooke CD, Faber TL, Tauxe EL, et al. Onset of left ventricular mechanical contraction as determined by phase analysis of ECG-gated myocardial perfusion SPECT imaging: Development of a diagnostic tool for assessment of cardiac mechanical dyssynchrony. J Nucl Cardiol 2005;12:68795.

4. Henneman MM, Chen J, Dibbets-Schneider P, Stokkel MP, Bleeker GB, Ypenburg C, et al. Can LV dyssynchrony as assessed with phase analysis on gated myocardial perfusion SPECT predict response to CRT? J Nucl Med 2007;48:1104-11.

5. Nakajima K, Okuda K, Matsuo S, Kiso K, Kinuya S, Garcia EV. Comparison of phase dyssynchrony analysis using gated myocardial perfusion imaging with four software programs: Based on the Japanese Society of Nuclear Medicine Working Group normal database. J Nucl Cardiol 2017;24:611-21.

6. Chen J, Garcia EV, Bax JJ, Iskandrian AE, Borges-Neto S, Soman P. SPECT myocardial perfusion imaging for the assessment of left ventricular mechanical dyssynchrony. J Nucl Cardiol 2011;18:685-94.

7. Trimble MA, Velazquez EJ, Adams GL, Honeycutt EF, Pagnanelli RA, Barnhart HX, et al. Repeatability and reproducibility of phase analysis of gated single-photon emission computed tomography myocardial perfusion imaging used to quantify cardiac dyssynchrony. Nucl Med Commun 2008;29:374-81.

8. AlJaroudi W, Jaber WA, Cerqueira MD. Effect of tracer dose on left ventricular mechanical dyssynchrony indices by phase analysis of gated single photon emission computed tomography myocardial perfusion imaging. J Nucl Cardiol 2012;19:63-72.

9. Barron AJ, Xavier R, Al-Housni M, Reyes E, Underwood R. Phase analysis, a novel SPECT technique for left ventricular dyssynchrony: Are degrees and milliseconds interchangeable? J Nucl Cardiol 2019. https://doi.org/10.1007/s12350-018-01574-y.

10. AlJaroudi W, Alraies MC, DiFilippo F, Brunken RC, Cerqueira MD, Jaber WA. Effect of stress testing on left ventricular mechanical synchrony by phase analysis of gated positron emission tomography in patients with normal myocardial perfusion. Eur J Nucl Med Mol Imaging 2012;39:665-72.

11. Chen CC, Shen TY, Chang MC, Hung GU, Chen WC, Kao CH, et al. Stress-induced myocardial ischemia is associated with early post-stress left ventricular mechanical dyssynchrony as assessed by phase analysis of ${ }^{201} \mathrm{Tl}$ gated SPECT myocardial perfusion imaging. Eur J Nucl Med Mol Imaging 2012;39:1904-9.
12. Singh H, Patel CD, Sharma P, Naik N, Singh S, Narang R. Does perfusion pattern influence stress-induced changes in left ventricular mechanical dyssynchrony on thallium-201-gated SPECT myocardial perfusion imaging? J Nucl Cardiol 2015;22:36-43.

13. Huang WS, Huang CH, Lee CL, Chen CP, Hung GU, Chen J. Relation of early post-stress left ventricular dyssynchrony and the extent of angiographic coronary artery disease. J Nucl Cardiol 2014;21:1048-56.

14. Hung GU, Chen CP, Yang KT. Incremental value of ischemic stunning on the detection of severe and extensive coronary artery disease in dipyridamole Tl-201 gated myocardial perfusion imaging. Int J Cardiol 2005;105:108-10.

15. Swett E, Massardo T, Hiplan E, Paillahueque G, Prat H. Intraventricular synchronism value for ischemia detection with Dipyridamole Sestamibi myocardial SPECT. In: Alasbimn Journal 2018 (XXVI Congreso ALASBIMN noviembre 2017, Santiago, Chile).

16. Aljaroudi W, Koneru J, Heo J, Iskandrian AE. Impact of ischemia on left ventricular dyssynchrony by phase analysis of gated single photon emission computed tomography myocardial perfusion imaging. J Nucl Cardiol 2011;18:36-42.

17. Van Kriekinge SD, Nishina H, Ohba M, Berman DS, Germano G. Automatic global and regional phase analysis from gated myocardial perfusion SPECT imaging: Application to the characterization of ventricular contraction in patients with left bundle branch block. J Nucl Med 2008;49:1790-7.

18. Takahashi N, Yamamoto A, Tezuka S, Ishikawa M, Abe J, Amitani $\mathrm{K}$, et al. Assessment of left ventricular dyssynchrony during development of heart failure by a novel program using ECG-gated myocardial perfusion SPECT. Circ J 2008;72:370-7.

19. AlJaroudi W, Jaber WA, Grimm RA, Marwick T, Cerqueira MD. Alternative methods for the assessment of mechanical dyssynchrony using phase analysis of gated single photon emission computed tomography myocardial perfusion imaging. Int J Cardiovasc Imaging 2012;28:1385-94.

20. Cooke CD, Esteves FP, Chen J, Garcia EV. Left ventricular mechanical synchrony from stress and rest ${ }^{82} \mathrm{Rb}$ PET myocardial perfusion ECG-gated studies: differentiating normal from LBBB patients. J Nucl Cardiol 2011;18:1076-85.

21. Peix A, Cabrera LO, Padron K, Rodriguez L, Fernandez J, Lopez $\mathrm{G}$, et al. Association between non-perfusion parameters and presence of ischemia in gated-SPECT myocardial perfusion imaging studies. J Nucl Cardiol 2018;25:609-15.

22. Hida S, Chikamori T, Tanaka H, Igarashi Y, Shiba C, Usui Y, et al. Diagnostic value of left ventricular dyssynchrony after exercise and at rest in the detection of multivessel coronary artery disease on single-photon emission computed tomography. Circ J 2012;76:1942-52.

23. Kerrigan J, Shivapour D, Tuzcu EM, Sabik EM, Jaber WA. Acute left ventricular dys-synchrony during regadenason PET myocardial perfusion stress test. J Nucl Cardiol 2015;22:388-90.

24. Massardo T, Gonzalez P, Humeres P, Chamorro H, Ayala F, Yovanovich J, et al. Simultaneous assessment of function and perfusion during dipyridamole-handgrip Tc-99m sestamibi imaging in chronic coronary artery disease. Ann Nucl Med 1999; 13:121-5.

25. Bestetti A, Cuko B, Decarli A, Galli A, Lombardi F. Additional value of systolic wall thickening in myocardial stunning evaluated by stress-rest gated perfusion SPECT. J Nucl Cardiol 2017. h ttps://doi.org/10.1007/s12350-017-1115-5.

26. Bestetti A, Cuko B, Gasparini M, De Servi S. Better characterization of dipyridamole-induced myocardial stunning by systolic wall thickening. A gated perfusion SPECT study. J Nucl Cardiol 2018. https://doi.org/10.1007/s12350-018-1340-6. 
27. Aggarwal H, AlJaroudi WA, Mehta S, Mannon R, Heo J, Iskandrian AE, et al. The prognostic value of left ventricular mechanical dyssynchrony using gated myocardial perfusion imaging in patients with end-stage renal disease. J Nucl Cardiol 2014;21:73946.

28. Hess PL, Shaw LK, Fudim M, Iskandrian AE, Borges-Neto S. The prognostic value of mechanical left ventricular dyssynchrony defined by phase analysis from gated single-photon emission computed tomography myocardial perfusion imaging among patients with coronary heart disease. J Nucl Cardiol 2017;24:48290.

29. Bajaj NS, Singh S, Farag A, El-Hajj S, Heo J, Iskandrian AE, et al. The prognostic value of non-perfusion variables obtained during vasodilator stress myocardial perfusion imaging. J Nucl Cardiol 2016;23:390-413.

Publisher's Note Springer Nature remains neutral with regard to jurisdictional claims in published maps and institutional affiliations. 